\title{
GENUS BOUNDS FOR CURVES WITH FIXED FROBENIUS EIGENVALUES
}

\author{
NOAM D. ELKIES, EVERETT W. HOWE, AND CHRISTOPHE RITZENTHALER
}

(Communicated by Ken Ono)

Abstract. For every finite collection $\mathcal{C}$ of abelian varieties over $\mathbf{F}_{q}$, we produce an explicit upper bound on the genus of curves over $\mathbf{F}_{q}$ whose Jacobians are isogenous to a product of powers of elements of $\mathcal{C}$.

Our explicit bound is expressed in terms of the Frobenius angles of the elements of $\mathcal{C}$. In general, suppose that $S$ is a finite collection of $s$ real numbers in the interval $[0, \pi]$. If $S=\{0\}$ set $r=1 / 2$; otherwise, let

$$
r=\#(S \cap\{\pi\})+2 \sum_{\theta \in S \backslash\{0, \pi\}}\left\lceil\frac{\pi}{2 \theta}\right\rceil .
$$

We show that if $C$ is a curve over $\mathbf{F}_{q}$ whose genus is greater than

$$
\min \left(23 s^{2} q^{2 s} \log q,(\sqrt{q}+1)^{2 r}\left(\frac{1+q^{-r}}{2}\right)\right),
$$

then $C$ has a Frobenius angle $\theta$ such that neither $\theta$ nor $-\theta$ lies in $S$.

We do not claim that this genus bound is best possible. For any particular set $S$ we can usually obtain a better bound by solving a linear programming problem. For example, we use linear programming to give a new proof of a result of Duursma and Enjalbert: If the Jacobian of a curve $C$ over $\mathbf{F}_{2}$ is isogenous to a product of elliptic curves over $\mathbf{F}_{2}$, then the genus of $C$ is at most 26. As Duursma and Enjalbert note, this bound is sharp, because there is an $\mathbf{F}_{2}$-rational model of the genus-26 modular curve $X(11)$ whose Jacobian splits completely into elliptic curves.

As an application of our results, we give a new proof of (and correct a small error in) a result of Yamauchi, which provides the complete list of positive integers $N$ such that the modular Jacobian $J_{0}(N)$ is isogenous over $\mathbf{Q}$ to a product of elliptic curves.

\section{INTRODUCTION}

Let $\left(C_{n}\right)$ be a sequence of curves over a finite field $k$ such that the genus of $C_{n}$ tends to infinity with $n$. Serre [15, Cor. 2, p. 93] applies a result of Tsfasman and Vlădut [18, 19] to show that the dimension of the largest $k$-simple isogeny factor of the Jacobian of $C_{n}$ tends to infinity with $n$. In this article we give an explicit bound for this asymptotic result.

The Weil polynomial of a $d$-dimensional abelian variety $A$ over a finite field $\mathbf{F}_{q}$ is the characteristic polynomial of the $q$-th power Frobenius endomorphism of $A$

Received by the editors February 28, 2012.

2010 Mathematics Subject Classification. Primary 14G10; Secondary 11G20, 14G15, 14 H25.

Key words and phrases. Curve, Jacobian, Weil polynomial, Frobenius eigenvalue, genus, linear programming.

The third author was partially supported by grant MTM2006-11391 from the Spanish MEC and by grant ANR-09-BLAN-0020-01 from the French ANR. 
(acting, for instance, on the $\ell$-adic Tate module of $A$ ). The Weil polynomial is a monic polynomial in $\mathbf{Z}[x]$ of degree $2 d$, and its complex roots $\alpha_{1}, \ldots, \alpha_{2 d}$ all have magnitude $\sqrt{q}$. The roots can be written $\alpha_{j}=\sqrt{q} \exp \left(i \theta_{j}\right)$ for real numbers $\theta_{j}$ in the half-open interval $(-\pi, \pi]$, and the roots can be ordered so that $\alpha_{j} \alpha_{j+d}=q$ and

$$
0 \leq \theta_{1} \leq \theta_{2} \leq \cdots \leq \theta_{d} \leq \pi
$$

The $\theta_{j}$ are called the Frobenius angles of $A$, and the $\theta_{j}$ that are nonnegative are the nonnegative Frobenius angles of $A$. If $C$ is a curve over $\mathbf{F}_{q}$, we define the Frobenius angles of $C$ to be the Frobenius angles of its Jacobian.

We show that for any finite set $S$ of real numbers in the interval $[0, \pi]$, every curve over $\mathbf{F}_{q}$ of sufficiently large genus has a nonnegative Frobenius angle that does not lie in $S$.

Theorem 1.1. Let $S$ be a finite set of s real numbers $\theta$ with $0 \leq \theta \leq \pi$. If $S=\{0\}$ set $r=1 / 2$; otherwise, take

$$
r=\#(S \cap\{\pi\})+2 \sum_{\theta \in S \backslash\{0, \pi\}}\left\lceil\frac{\pi}{2 \theta}\right\rceil,
$$

where $\lceil x\rceil$ denotes the least integer greater than or equal to the real number $x$. Let

$$
B_{1}=23 s^{2} q^{2 s} \log q \quad \text { and } \quad B_{2}=(\sqrt{q}+1)^{2 r}\left(\frac{1+q^{-r}}{2}\right) .
$$

If $C$ is a curve over $\mathbf{F}_{q}$ whose nonnegative Frobenius angles all lie in $S$, then the genus $g$ of $C$ satisfies $g \leq B_{1}$ and $g<B_{2}$.

Remark 1.2. Elementary calculations show that if $s>7 \sqrt{q} \log q$, then the bound $B_{1}$ from Theorem 1.1 is smaller than the bound $B_{2}$.

Theorem 1.1 allows us to derive an explicit version of Serre's result.

Corollary 1.3. If $C$ is a curve of genus $g>2$ over a finite field $\mathbf{F}_{q}$, then the Jacobian of $C$ has a simple isogeny factor of dimension greater than

$$
\sqrt{\frac{\log \log g}{6 \log q}}
$$

The first genus bound from Theorem 1.1 leads to a corollary that does not mention Frobenius angles.

Corollary 1.4. Let $A$ be a d-dimensional abelian variety over $\mathbf{F}_{q}$. If $C$ is a curve over $\mathbf{F}_{q}$ of genus greater than $23 d^{2} q^{2 d} \log q$, then the Jacobian of $C$ has a simple isogeny factor $B$ that is not an isogeny factor of $A$.

One natural choice for the set $S$ in Theorem 1.1 is the set of all nonnegative Frobenius angles for elliptic curves over a given finite field. Applying the theorem to this set leads to the following corollary:

Corollary 1.5. Suppose $C$ is a curve over $\mathbf{F}_{q}$ whose Jacobian is isogenous over $\mathbf{F}_{q}$ to a product of elliptic curves. Then the genus of $C$ is at most $510 q^{8 \sqrt{q}+3} \log q$.

We prove Theorem 1.1 and its corollaries in Section 2 As we will show in Remark 2.6. for every prime power $q$ there is a family of sets $S$ for which (in the notation of Theorem 1.1) the ratio $B_{1} / g$ is less than $47 s^{2} \log q$. Similarly, in Remark 2.4 we show that there is a family of examples with $s=1$ and $q \rightarrow \infty$ 
for which the ratio $B_{2} / g$ approaches 1 . For many sets $S$, however, we expect that the bounds given by Theorem 1.1 are likely to be far from optimal; in Section 3 we show how tighter bounds can sometimes be obtained by solving an integer linear programming problem. As a concrete example, we take $S$ to be the set of nonnegative Frobenius angles of the elliptic curves over $\mathbf{F}_{2}$ and use the linear programming method to show that the genus of a curve over $\mathbf{F}_{2}$ whose Jacobian splits up to isogeny into elliptic curves is at most 26, a result proved earlier by Duursma and Enjalbert 8 by a different (but related) method. In Section 4 we show that for this particular set $S$ the genus bound of 26 is sharp, because the genus-26 modular curve $X(11)$ has a model over $\mathbf{F}_{2}$ whose Jacobian is isogenous over $\mathbf{F}_{2}$ to a product of elliptic curves. (We find sharp upper bounds in other cases as well; see Remark [3.7.) In Section [5 we use this genus bound to give a simple proof of a result of Yamauchi 21] on the values of $N$ for which the Jacobian of the modular curve $X_{0}(N)$ is isogenous over $\mathbf{Q}$ to a product of elliptic curves.

Conventions and notation. Operators such as Hom, End or Aut applied to varieties over a field $k$ will always refer to $k$-rational homomorphisms and endomorphisms. Similarly, when we say that an abelian variety over $k$ is 'decomposable', 'split', or 'simple', these words should be interpreted with respect to isogenies over $k$. In the sequel, we use $e(x)$ to denote $\exp (i x)$, where $i=\sqrt{-1}$, and we use $\operatorname{Re}(x)$ to denote the real part of a complex number $x$.

\section{Proof of Theorem 1.1 and its Corollaries}

In this section we prove Theorem 1.1 and Corollaries 1.4 and 1.5. The arguments that show that $B_{1}$ and $B_{2}$ give upper bounds on the genus are independent of one another, so we break the proof of Theorem 1.1 into two parts.

Proof of Theorem 1.1, part 1: $g \leq B_{1}$. The bound $g \leq B_{1}$ holds trivially when $S$ is empty, so we may assume that $s>0$. First we consider the case where $s>1$.

Suppose $C$ is a curve over $\mathbf{F}_{q}$ all of whose Frobenius angles lie in $S$. Let the elements of $S$ be $\theta_{1}, \ldots, \theta_{s}$, and for each index $j$ let $b_{j}$ denote the multiplicity of $\theta_{j}$ as a Frobenius angle for $C$ (unless $\theta_{j}=0$ or $\theta_{j}=\pi$, in which case we let $b_{j}$ denote half of this multiplicity) so that we have

$$
b_{1}+\ldots+b_{s}=g .
$$

Reindex the $\theta_{j}$ and the corresponding $b_{j}$ so that $b_{1} \geq b_{2} \geq \cdots \geq b_{s}$, and note that this implies that $b_{1} \geq g / s$. Finally, for every $j$ let $z_{j}=e\left(\theta_{j}\right)$, and for every integer $k>0$ let $G(k)$ denote the weighted power sum

$$
G(k)=b_{1} z_{1}^{k}+\cdots+b_{s} z_{s}^{k} .
$$

Weil's 'Riemann Hypothesis' for curves over finite fields says that

$$
\# C\left(\mathbf{F}_{q^{k}}\right)=q^{k}+1-\sum_{j=1}^{2 g} \alpha_{j}^{k}=q^{k}+1-2 q^{k / 2} \operatorname{Re} G(k),
$$

so we find that

$$
2 \operatorname{Re} G(k)=q^{k / 2}+q^{-k / 2}-\# C\left(\mathbf{F}_{q^{k}}\right) / q^{k / 2} .
$$

Now we apply a result of Smyth [16]. Let

$$
\beta:=\left(b_{2}+\cdots+b_{s}\right) / b_{1},
$$


let $\lambda$ be an arbitrary real number with $0<\lambda \leq 1$, and let

$$
K:=1+\lfloor(4 \beta+3) / \lambda\rfloor .
$$

Smyth shows that then

$$
\left(b_{1} / 4\right)(1-\lambda)<\max _{1 \leq k \leq K} \operatorname{Re} G(k) .
$$

In our case, we have $\beta=g / b_{1}-1 \leq s-1$, so $K \leq 1+(4 \beta+3) / \lambda \leq L$, where we set

$$
L:=1+(4 s-1) / \lambda .
$$

Also, for each $k$ we have $\operatorname{Re} G(k) \leq(1 / 2)\left(q^{k / 2}+q^{-k / 2}\right)$. Since $q^{x}+q^{-x}$ is an increasing function of $x$ for $x>0$, Smyth's result shows that

$$
\left(b_{1} / 4\right)(1-\lambda)<(1 / 2)\left(q^{L / 2}+q^{-L / 2}\right) .
$$

As we noted earlier, we have $b_{1} \geq g / s$, so for every $\lambda$ with $0<\lambda<1$ we have

$$
\frac{g}{s}<\frac{2}{1-\lambda}\left(q^{L / 2}+q^{-L / 2}\right)
$$

We will apply this inequality with $\lambda=1-1 / a$, where

$$
a=\left(\frac{4 s-1}{2}\right) \log q .
$$

(This choice of $\lambda$ was obtained by taking the first two terms of the power series expansion (in $1 / a$ ) of the value of $\lambda$ that minimizes $q^{L / 2} /(1-\lambda)$.) Note that $L>4 s \geq 8$ so that

$$
q^{L / 2}+q^{-L / 2}=q^{L / 2}\left(1+q^{-L}\right)<\frac{257}{256} q^{L / 2} .
$$

Combining this with (1), we obtain

$$
\begin{aligned}
\frac{g}{s} & <\left(\frac{257}{256}\right)\left(\frac{2}{1-\lambda}\right) q^{L / 2} \\
& =\left(\frac{257}{256}\right)(4 s-1)(\log q) \exp ((L / 2) \log q) .
\end{aligned}
$$

Using the equality $L=1+(4 s-1) / \lambda$, we find that

$$
\begin{aligned}
(L / 2) \log q & =(\log q) / 2+\left(\frac{4 s-1}{2}\right) \frac{\log q}{\lambda} \\
& =(\log q) / 2+a / \lambda \\
& =(\log q) / 2+a^{2} /(a-1) \\
& =(\log q) / 2+a+a /(a-1) \\
& =2 s \log q+a /(a-1) .
\end{aligned}
$$

Since $s \geq 2$ and $q \geq 2$ we have $a \geq(7 / 2) \log 2$ and $\exp (a /(a-1))<5$.5. Thus

$$
\exp ((L / 2) \log q)<5.5 \exp (2 s \log q)=5.5 q^{2 s},
$$

so

$$
\frac{g}{s}<\left(\frac{257}{256}\right)(4 s)(\log q)\left(5.5 q^{2 s}\right)<23 s q^{2 s} \log q .
$$

This shows that $g<B_{1}$ when $s>1$. 
Now suppose $s=1$. Let $\theta$ be the unique element of $S$. If $\theta \geq \pi / 2$, then the quantity $r$ defined in the statement of the theorem is equal to 2 . It is easy to show that then $B_{2}<B_{1}$, so the bound $g<B_{2}$, proved below, shows that $g<B_{1}$.

The final case to consider is when $s=1$ and $\theta<\pi / 2$. If $C$ has only one nonnegative Frobenius angle $\theta$, then $e(\theta) \sqrt{q}$ must be an algebraic integer of degree at most 2 over the rationals, so the quantity $t=e(\theta) \sqrt{q}+e(-\theta) \sqrt{q}$ is an integer. Furthermore, since $\theta$ is less than $\pi / 2$, the integer $t$ is positive. Then Weil's theorem shows that

$$
0 \leq \# C\left(\mathbf{F}_{q}\right)=q+1-g t \leq q+1-g
$$

so that $g \leq q+1$. Again, it follows easily that $g<B_{1}$.

Our proof that $B_{2}$ gives an upper bound for the genus relies upon the following lemma.

Lemma 2.1. Let $S$ be a set of real numbers in $[0, \pi]$ and let $T \in \mathbf{R}[X]$ be a polynomial with nonnegative coefficients such that $T(0)=0$ and $\operatorname{Re}(T(e(\theta))) \geq 1$ for all $\theta \in S$. If $C$ is a curve over $\mathbf{F}_{q}$ whose nonnegative Frobenius angles all lie in $S$, then the genus of $C$ is at most $\left(T\left(q^{1 / 2}\right)+T\left(q^{-1 / 2}\right)\right) / 2$.

Proof. Let $\alpha_{1}, \ldots, \alpha_{2 g}$ be the complex roots of the Weil polynomial of $C$, listed with appropriate multiplicities, so that from Weil's theorem we have

$$
\# C\left(\mathbf{F}_{q^{m}}\right)=q^{m}+1-\sum_{j=1}^{2 g} \alpha_{j}^{m}
$$

for all integers $m>0$. Write

$$
T=a_{1} x+\cdots+a_{n} x^{n}, \quad a_{j} \geq 0 .
$$

Then

$$
\begin{aligned}
0 & \leq \sum_{m=1}^{n} a_{m} \frac{\# C\left(\mathbf{F}_{q^{m}}\right)}{q^{m / 2}} \\
& =\sum_{m=1}^{n} a_{m}\left(q^{m / 2}+q^{-m / 2}-\sum_{j=1}^{2 g} \alpha_{j}^{m} / q^{m / 2}\right) \\
& =T\left(q^{1 / 2}\right)+T\left(q^{-1 / 2}\right)-\sum_{j=1}^{2 g} T\left(\alpha_{j} / \sqrt{q}\right) .
\end{aligned}
$$

By hypothesis, each summand $T\left(\alpha_{j} / \sqrt{q}\right)$ has real part at least 1 , so

$$
0 \leq T\left(q^{1 / 2}\right)+T\left(q^{-1 / 2}\right)-2 g,
$$

and hence

$$
g \leq \frac{T\left(q^{1 / 2}\right)+T\left(q^{-1 / 2}\right)}{2}
$$

Remark 2.2. This lemma could also be proved by using the results in $\S 2.4$ of the arXiv version of [8].

Remark 2.3. Lemma 2.1 is stated for polynomials $T$ with nonnegative coefficients, but an analogous statement holds when $T$ is a power series in $\mathbf{R}[[x]]$ with nonnegative coefficients, so long as its radius of convergence exceeds $\sqrt{q}$. 
With this lemma in hand, we complete the proof of Theorem 1.1. Our proof will depend on a careful choice of the polynomial $T$.

Proof of Theorem 1.1, part 2: $g<B_{2}$. It is easy to check that $g<B_{2}$ when $S$ is empty, so we may assume that $S$ is nonempty. If $S=\{0\}$, then the quantity $r$ from the theorem is equal to $1 / 2$. In this case we can take $T=x$, and we find that $\left(T\left(q^{1 / 2}\right)+T\left(q^{-1 / 2}\right)\right) / 2$ is less than $B_{2}$. Then Lemma 2.1 shows that $g<B_{2}$. So let us assume that $S$ contains a nonzero element.

Given a nonzero $\theta \in S$, let $m=\left\lceil\frac{\pi}{2 \theta}\right\rceil$ so that $\cos (m \theta) \leq 0$. If $\theta=\pi$ let $P_{\theta}$ be the polynomial $1+x$; otherwise, set $P_{\theta}=1-2 \cos (m \theta) x^{m}+x^{2 m}$. In both cases we have $P_{\theta}(e(\theta))=0$. Let

$$
P=\prod_{\theta \in S \backslash\{0\}} P_{\theta}
$$

Then $P$ is a polynomial with constant term 1 , with nonnegative coefficients, and with degree equal to $r$, where

$$
r=\#(S \cap\{\pi\})+2 \sum_{\theta \in S \backslash\{0, \pi\}}\left\lceil\frac{\pi}{2 \theta}\right\rceil
$$

is as in the statement of the theorem. Let $T=(P-1)^{2}$. Then $T(0)=0$, the coefficients of $T$ are nonnegative, and for every $\theta \in S \backslash\{0\}$ we have $T(e(\theta))=1$. Also, for each $\theta$ we have $P_{\theta}(1) \geq 2$, so $P(1) \geq 2$ and $T(1) \geq 1$. Lemma 2.1 shows that any curve whose nonnegative Frobenius angles all lie in $S$ must have genus no larger than $\left(T\left(q^{1 / 2}\right)+T\left(q^{-1 / 2}\right)\right) / 2$. Now, for positive real numbers $z$ we have $1 \leq P(z) \leq(1+z)^{r}$ so that $T(z)<(1+z)^{2 r}$. Thus we have

$$
\begin{aligned}
T\left(q^{1 / 2}\right)+T\left(q^{-1 / 2}\right) & <(\sqrt{q}+1)^{2 r}+(1 / \sqrt{q}+1)^{2 r} \\
& =(\sqrt{q}+1)^{2 r}\left(1+q^{-r}\right),
\end{aligned}
$$

which gives the inequality $g<B_{2}$.

Remark 2.4. In Section 3 we will see that our bounds can sometimes be bad. However, the following easy example shows that at least in one case our second bound is asymptotically exact as $q \rightarrow \infty$. For any prime power $q$ let $E$ be a supersingular elliptic over $\mathbf{F}_{q}$ with Weil polynomial $x^{2}+q$, corresponding to the set $S=\{\pi / 2\}$. Applying Theorem 1.1, we see that the genus of a curve $C / \mathbf{F}_{q}$ with Jacobian isogenous to a power of $E$ is bounded above by

$$
(\sqrt{q}+1)^{4} \cdot\left(\frac{1+q^{-2}}{2}\right) \sim q^{2} / 2 \quad \text { as } q \rightarrow \infty .
$$

On the other hand, $\operatorname{Jac}(C)$ is isogenous to a power of $E$ if and only if $C$ is optimal over $\mathbf{F}_{q^{2}}$, in the sense that its number of points attains the Weil upper bound. But the maximal genus of such a curve is $q(q-1) / 2 \sim q^{2} / 2$ (see [11]), attained by the Hermitian curve $H_{q}$ defined by $x^{q+1}+y^{q+1}+z^{q+1}=0$ (see for instance [14]).

Remark 2.5. The Hermitian curve $H_{q}$, viewed as a curve over $\mathbf{F}_{q^{2}}$, again gives an example of a curve whose genus comes close to the upper bound $B_{2}$. If we take $S=\{\pi\}$, then the bound $B_{2}$ for the field $\mathbf{F}_{q^{2}}$ is

$$
(q+1)^{2} \cdot\left(\frac{1+q^{-2}}{2}\right) \sim q^{2} / 2 \quad \text { as } q \rightarrow \infty,
$$

while $H_{q}$ has genus $q(q-1) / 2$. 
Remark 2.6. Hermitian curves can also be used to give examples that limit the extent to which we might hope to improve the bound $B_{1}$. For any integer $s>0$, let $S$ be the $s$-element set

$$
S=\left\{\frac{\pi}{2 s}, \frac{3 \pi}{2 s}, \ldots, \frac{(2 s-1) \pi}{2 s}\right\} .
$$

Let $q$ be any prime power and set $Q=q^{s}$. Note that the nonnegative Frobenius angles of a curve $C$ over $\mathbf{F}_{q}$ are contained in $S$ if and only if the only nonnegative Frobenius angle of the base extension of $C$ to $\mathbf{F}_{Q}$ is $\pi / 2$. Let $C$ be the curve $x^{Q+1}+y^{Q+1}+z^{Q+1}=0$ over $\mathbf{F}_{q}$. As noted in Remark 2.4 the base extension of $C$ to $\mathbf{F}_{Q}$ has $\pi / 2$ as its unique Frobenius angle, so all of the Frobenius angles of $C$ itself are contained in $S$. Since the genus of $C$ is $\left(q^{2 s}-q^{s}\right) / 2$, we see that Theorem 1.1 would be false if we replaced $B_{1}$ with any expression of the form

$$
\text { (polynomial in } s \text { and } \log q) q^{2 s-\varepsilon}
$$

for a positive constant $\varepsilon$.

We end this section by proving the corollaries from the introduction.

Proof of Corollary 1.3. We begin by noting that for every integer $n>0$, the number of isogeny classes of $n$-dimensional abelian varieties over $\mathbf{F}_{q}$ is less than $6^{n^{2}} q^{n(n+1) / 4}$. This is easy to check for $n=1$ (see the proof of Corollary 1.5 below) and for $n=2$, while for $n>2$ it follows from the results in [6] (as corrected in [7]). We leave the details of the argument to the reader, but we do at least note that it is helpful to observe that for every $n$, the quantity $v_{n}$ in [6, Thm. 1.2] is bounded above by 264. It follows that the number of isogeny classes of simple abelian varieties over $\mathbf{F}_{q}$ of dimension at most $n$ is also less than $6^{n^{2}} q^{n(n+1) / 4}$ and that the number of nonnegative Frobenius angles of abelian varieties over $\mathbf{F}_{q}$ of dimension at most $n$ is less than $n 6^{n^{2}} q^{n(n+1) / 4}$.

Suppose, to obtain a contradiction, that the corollary is false, and let $C$ be a curve of genus $g$ over $\mathbf{F}_{q}$ that provides a counterexample. Take

$$
n=\left\lfloor\sqrt{\frac{\log \log g}{6 \log q}}\right\rfloor .
$$

For $C$ to provide a counterexample we must have $n \geq 1$. We will apply Theorem 1.1 to the set of nonnegative Frobenius angles of abelian varieties over $\mathbf{F}_{q}$ of dimension at most $n$. As we have just noted, this means that in Theorem 1.1] we have $s<n 6^{n^{2}} q^{n(n+1) / 4}$. Simple estimates for the terms in the right-hand side of this inequality show that

$$
s<q^{4 n^{2}} .
$$

Note that there are at least 5 isogeny classes of elliptic curves over any field, so $s \geq 5$; using this fact it is easy to show that the bound $B_{1}=23 s^{2} q^{2 s} \log q$ from the theorem satisfies

$$
B_{1}<q^{4 s}
$$

The definition of $n$ tells us that

$$
\log \log g \geq 6 n^{2} \log q
$$


so exponentiating gives us

$$
\log g \geq q^{6 n^{2}}>4 q^{4 n^{2}} \log q>4 s \log q,
$$

where the third inequality follows from (2). Exponentiating again, we find that

$$
g>q^{4 s}>B_{1},
$$

by (3). Theorem 1.1 then shows that Jac $C$ has a simple isogeny factor of dimension greater than $n$, contradicting our assumption that $C$ was a counterexample to the corollary. This contradiction completes the proof.

Proof of Corollary 1.4. Let $S$ be the set of nonnegative Frobenius angles of the abelian variety $A$ over $\mathbf{F}_{q}$ so that $\# S \leq d$. If $C$ is a curve over $\mathbf{F}_{q}$ with genus greater than $23 d^{2} q^{2 d} \log q$, then $C$ has a nonnegative Frobenius angle $\theta$ not in $S$. Let $B$ be any element of the unique isogeny class of simple abelian varieties over $\mathbf{F}_{q}$ that have $\theta$ as one of their Frobenius angles. Then $B$ is an isogeny factor of Jac $C$. On the other hand, $A$ and $B$ have coprime Weil polynomials, so by the Honda-Tate theorem $B$ is not an isogeny factor of $A$.

Proof of Corollary 1.5. Let $m$ be the largest integer with $m^{2} \leq 4 q$. The Weil polynomial of an elliptic curve over $\mathbf{F}_{q}$ is of the form $x^{2}-t x+q$, where $t$ is an integer with $-m \leq t \leq m$. (Not every $t$ in this range need come from an elliptic curve.) The nonnegative Frobenius angle $\theta_{t}$ corresponding to a given $t$ is $\theta_{t}=\cos ^{-1}(t /(2 \sqrt{q}))$. We take $S$ to be the set $\left\{\theta_{t}:-m \leq t \leq m\right\}$.

Clearly $\# S \leq 4 \sqrt{q}+1$. The bound $B_{1}$ from Theorem 1.1 is then

$$
\begin{aligned}
23 s^{2} q^{2 s} \log q & \leq 23(4 \sqrt{q}+1)^{2} q^{8 \sqrt{q}+2} \log q \\
& \leq 23\left(\frac{4 \sqrt{2}+1}{\sqrt{2}}\right)^{2} q^{8 \sqrt{q}+3} \log q \\
& <510 q^{8 \sqrt{q}+3} \log q .
\end{aligned}
$$

\section{LINEAR PROGRAMMING}

The arguments we used to prove Theorem 1.1 used no special properties of the set $S$ and give a bound that is almost certainly far from optimal when applied to most $S$. For example, suppose we take $S$ to be the set of nonnegative Frobenius angles coming from the elliptic curves over $\mathbf{F}_{2}$. There are five isogeny classes of elliptic curves over $\mathbf{F}_{2}$ (each containing a single curve), corresponding to the five possible traces of Frobenius -2, $-1,0,1,2$ (see [5], 20, Theorem 4.1]). For each of these traces $t$, let $\alpha_{t}=\left(-t+\sqrt{t^{2}-8}\right) / 2$, let $\theta_{t}$ be the argument of $\alpha_{t}$, and let $E_{t}$ be an elliptic curve over $\mathbf{F}_{2}$ with trace $t$. Let us apply Theorem 1.1 to the set $S=\left\{\theta_{t}\right\}$. We find that $B_{1} \approx 408125$ and $B_{2} \approx 2.6 \times 10^{10}$. However, the best upper bound on the genus is much smaller than these numbers.

Theorem 3.1 (Duursma and Enjalbert [8]). Suppose $C$ is a curve over $\mathbf{F}_{2}$ whose Jacobian is isogenous to a product of powers of the $E_{t}$. Then the genus of $C$ is at most 26.

Remark 3.2. Duursma and Enjalbert prove this result by using a stronger version of our Lemma 2.1. We give the proof presented here as an example of our general technique of using linear programming to get genus bounds. 
Remark 3.3. We will see in Section 4 that the bound in Theorem 3.1 is sharp.

Proof of Theorem 3.1. Our bounds in Theorem 1.1 were obtained from the fact that the number of points on a curve over a finite field is always nonnegative. We actually know a somewhat stronger constraint: For every $n>0$, the number of degree- $n$ places on a curve is nonnegative. If the size of the base field is large compared to the genus of the curve in question, the bounds we get from using place counts are not much better than the one we get from point counts. However, Theorem 3.1 involves a very small field indeed.

Let $C$ be a curve as in the statement of the theorem. In particular, suppose there are integers $e_{t} \geq 0$ such that the Jacobian of $C$ is isogenous to

$$
E_{-2}^{e_{-2}} \times E_{-1}^{e_{-1}} \times E_{0}^{e_{0}} \times E_{1}^{e_{1}} \times E_{2}^{e_{2}} .
$$

Then for every $n>0$ the number of points on $C$ over $\mathbf{F}_{2^{n}}$ is given by

$$
\# C\left(\mathbf{F}_{2^{n}}\right)=2^{n}+1-\sum_{-2 \leq t \leq 2} e_{t} \operatorname{Tr}\left(\alpha_{t}^{n}\right),
$$

and the number $N_{n}$ of degree- $n$ places on $C$ is given by

$$
N_{n}=\frac{1}{n} \sum_{d \mid n} \mu(n / d) \# C\left(\mathbf{F}_{2^{d}}\right),
$$

where $\mu$ is the Möbius function.

The nonnegativity of the number of degree- $n$ places, for $n=1, \ldots, 8$, is expressed by the following inequalities:

$$
\begin{array}{rrrrrl}
-2 e_{-2} & -e_{-1} & & +e_{1} & +2 e_{2} & \leq 3 \\
e_{-2} & -e_{-1} & -2 e_{0} & -2 e_{1} & -e_{2} & \leq 1 \\
2 e_{-2} & +2 e_{-1} & & -2 e_{1} & -2 e_{2} & \leq 2 \\
-2 e_{-2} & +e_{-1} & +3 e_{0} & +e_{1} & -2 e_{2} & \leq 3 \\
2 e_{-2} & -2 e_{-1} & & +2 e_{1} & -2 e_{2} & \leq 6 \\
-e_{-2} & +e_{-1} & -2 e_{0} & +3 e_{1} & +e_{2} & \leq 9 \\
-2 e_{-2} & +2 e_{-1} & & -2 e_{1} & +2 e_{2} & \leq 18 \\
5 e_{-2} & -4 e_{-1} & +3 e_{0} & -4 e_{1} & +5 e_{2} & \leq 30 .
\end{array}
$$

We claim that $\sum_{t=-2}^{2} e_{t} \leq 26$ for any nonnegative integers $e_{t}$ satisfying the inequalities above. Indeed we show this even if the $e_{t}$ are allowed to be nonnegative real numbers. Maximizing their sum is then a linear programming problem. Solving the dual problem, we find that if we take 39 times the third inequality of (4), plus 44 times the fourth inequality, plus 78 times the sixth inequality, plus 32 times the eighth inequality, we obtain

$$
72 e_{-2}+72 e_{-1}+72 e_{0}+72 e_{1}+72 e_{2} \leq 1872,
$$

so that $e_{-2}+e_{-1}+e_{0}+e_{1}+e_{2} \leq 1872 / 72=26$, as claimed.

Remark 3.4. By enumerating 5-tuples of nonnegative integers $\left(e_{-2}, e_{-1}, e_{0}, e_{1}, e_{2}\right)$ that sum to 26 and checking whether they satisfy the inequalities (44), one finds that if a genus-26 curve over $\mathbf{F}_{2}$ has a completely split Jacobian, then the Jacobian 
is isogenous to one of the varieties

$$
\begin{aligned}
& E_{-2}^{4} \times E_{-1}^{7} \times E_{0}^{5} \times E_{1}^{3} \times E_{2}^{7}, \\
& E_{-2}^{5} \times E_{-1}^{6} \times E_{0}^{5} \times E_{1}^{4} \times E_{2}^{6}, \text { or } \\
& E_{-2}^{6} \times E_{-1}^{5} \times E_{0}^{5} \times E_{1}^{5} \times E_{2}^{5} .
\end{aligned}
$$

Remark 3.5. We would not get a genus bound if we looked only at the inequalities coming from counting the places of degree strictly less than 8 . The reader can check that for any even $g$, the values $e_{-2}=e_{2}=g / 2$ and $e_{-1}=e_{0}=e_{1}=0$ satisfy the first seven inequalities in the system (41). On the other hand, a priori there is no reason to think that the bound we get from looking at the counts of places of degree 8 and less will be the best possible. Perhaps by using the nonnegativity of several more place counts, we would get a better bound. In this particular instance, results of Section 4 show that the bound we obtain is in fact sharp.

Remark 3.6. A similar argument, using places of degree at most 12, shows that a curve over $\mathbf{F}_{3}$ whose Jacobian is isogenous to a product of elliptic curves must have genus less than or equal to 2091. By extending the argument slightly (or by using an integer linear programming package, such as the one in Magma [2]) one can improve this upper bound to 2085. We suspect that this bound is not sharp!

Remark 3.7. If one restricts to curves whose Jacobians are isogenous to a product of powers of ordinary elliptic curves, one finds a genus bound of 3 over $\mathbf{F}_{2}$ and 26 over $\mathbf{F}_{3}$. The first bound is reached by the curve

$$
x^{4}+y^{4}+z^{4}+x^{2} y^{2}+x^{2} z^{2}+y^{2} z^{2}+x^{2} y z+x y^{2} z+x y z^{2}=0,
$$

which is a twist of the Klein quartic; the second bound is reached as well, as we show in Section 4 .

\section{The Modular CuRve $X(11)$}

In this section we show that the modular curve $X(11)$ has a model defined over $\mathbf{F}_{2}$ whose Jacobian is isogenous over $\mathbf{F}_{2}$ to a product of elliptic curves. Since $X(11)$ has genus 26, this example shows that the bound of Theorem 3.1 is sharp. Duursma and Enjalbert [8] provide a different proof that $X(11)$ has a model over $\mathbf{F}_{2}$ with completely split Jacobian. Their argument, found in the addendum to the arXiv version of their paper, relies on working with Klein's explicit model of $X(11)$ in $\mathbf{P}^{4}$.

Let $G$ be the twist of the finite group scheme $\mathbf{Z} / 11 \mathbf{Z}$ over $\mathbf{F}_{2}$ on which the $\mathbf{F}_{2^{-}}$ Frobenius acts as multiplication by 3 , and let $G^{\prime}$ be the Cartier dual of $G$. We see that $G^{\prime}$ is the twist of $\mathbf{Z} / 11 \mathbf{Z}$ on which the $\mathbf{F}_{2}$-Frobenius acts as multiplication by $2 / 3=-3 \bmod 11$. Let $e: G \times G^{\prime} \rightarrow \mathbf{G}_{m}$ be the natural pairing from $G \times G^{\prime}$ to the multiplicative group.

Let $X$ be the modular curve over $\mathbf{F}_{2}$ whose noncuspidal $K$-rational points, for every extension $K$ of $\mathbf{F}_{2}$, parametrize pairs $(E, \varphi)$, where $E$ is an elliptic curve over $K$ and $\varphi$ is an isomorphism from the group scheme $E[11]$ to the group scheme $\left(G \times G^{\prime}\right) \otimes_{\mathbf{F}_{2}} K$ that takes the Weil pairing on $E[11]$ to the pairing $e$.

Theorem 4.1. The genus of $X$ is 26, and the Jacobian of $X$ is isogenous to

$$
E_{-2}^{6} \times E_{-1}^{5} \times E_{0}^{5} \times E_{1}^{5} \times E_{2}^{5},
$$

where each $E_{t}$ is an elliptic curve over $\mathbf{F}_{2}$ with trace $t$. 
Proof. The curve $X$ is geometrically isomorphic to $X(11)$, so it has genus 26 and geometric automorphism group isomorphic to $\mathrm{PSL}_{2}(11)$ (see [13, Théorème 5] and [1]). Consider the group scheme $\left(G \times G^{\prime}\right) \otimes_{\mathbf{F}_{2}} \mathbf{F}_{4}$; it is simply $(\mathbf{Z} / 11 \mathbf{Z})^{2}$, with the $\mathbf{F}_{4}$-Frobenius acting as multiplication by -2 . The automorphism group of this $\mathbf{F}_{4^{-}}$ scheme is $\mathrm{GL}_{2}(11)$, and the subgroup of automorphisms that respect the pairing $e$ is isomorphic to $\mathrm{SL}_{2}(11)$. There is a surjective map

$$
\operatorname{Aut}\left(\left(G \times G^{\prime}\right) \otimes_{\mathbf{F}_{2}} \mathbf{F}_{4}, e\right) \rightarrow \operatorname{Aut}\left(X \otimes_{\mathbf{F}_{2}} \mathbf{F}_{4}\right)
$$

that sends an automorphism $\alpha$ of the finite group scheme to the automorphism $\beta$ of $X \otimes_{\mathbf{F}_{2}} \mathbf{F}_{4}$ that takes a pair $(E, \varphi)$ to $(E, \alpha \varphi)$, and the kernel of this map is the group $\{ \pm 1\}$. Therefore all of the geometric automorphisms of $X$ are already defined over $\mathbf{F}_{4}$.

Using [10, Lemma 2.1] we see that the twists of the curve $X \otimes \mathbf{F}_{2} \mathbf{F}_{4}$ correspond to the conjugacy classes of $\operatorname{Aut}\left(X \otimes_{\mathbf{F}_{2}} \mathbf{F}_{4}\right)$. Also, the automorphism group of the twist corresponding to the conjugacy class of an element $\alpha$ is isomorphic to the commutator of $\alpha$. Since $\mathrm{PSL}_{2}(11)$ has trivial center, we see that every nontrivial twist of $X \otimes_{\mathbf{F}_{2}} \mathbf{F}_{4}$ has an automorphism group strictly smaller than $\mathrm{PSL}_{2}(11)$.

Now take the $\mathbf{Q}(\sqrt{-11})$-rational model $Y$ of $X(11)$ considered by Ligozat 12 , Example 3.7.3, pp. 199-200]; Ligozat calls this curve $X(11)_{K_{11}}$. Let $\mathfrak{p}$ be the prime of $\mathbf{Q}(\sqrt{-11})$ over 2 , with residue field $\mathbf{F}_{4}$. The automorphism group of $Y$ is $\mathrm{PSL}_{2}(11)$, so the reduction of $Y$ modulo $\mathfrak{p}$ also has automorphism group $\mathrm{PSL}_{2}$ (11). Therefore, the reduction of $Y$ must be $X \otimes \mathbf{F}_{2} \mathbf{F}_{4}$. Applying a result of Ligozat [12, Prop 3.6.1, p. 223], we find that the Jacobian of $X$ is isogenous to $E_{-2}^{11} \times E_{0}^{5} \times E_{1}^{10}$. (To see this, we must take the elliptic curves mentioned in Ligozat's proposition and compute their reductions modulo $\mathfrak{p}$.)

It follows that over $\mathbf{F}_{2}$, the Jacobian of $X$ is isogenous to

$$
E_{-2}^{a} E_{-1}^{b} E_{0}^{5} E_{1}^{10-b} E_{2}^{11-a}
$$

for some choice of $a$ and $b$, and by Remark 3.4, we know that $(a, b)$ is one of $(4,7)$, $(5,6)$, or $(6,5)$.

For each of the three possible isogeny classes we can compute the associated zeta function and the number of $\mathbf{F}_{2}$-rational points on a curve with that zeta function. We find that if the number of $\mathbf{F}_{2}$-rational points on $X$ is 1 , then $(a, b)=(4,7)$; if $\# X\left(\mathbf{F}_{2}\right)=3$, then $(a, b)=(5,6)$; and if $\# X\left(\mathbf{F}_{2}\right)=5$, then $(a, b)=(6,5)$.

Consider $E_{0}$, the unique elliptic curve over $\mathbf{F}_{2}$ with trace 0 . The characteristic polynomial of Frobenius on $E_{0}$ [11] is $x^{2}+2$, so the action of Frobenius on $E_{0}$ [11] has two eigenspaces, one with eigenvalue 3 and one with eigenvalue -3 . One finds that there are 10 pairing-respecting isomorphisms $\varphi: E_{0}[11] \rightarrow G \times G^{\prime}$, and since $\left(E_{0}, \varphi\right)$ and $\left(E_{0},-\varphi\right)$ are represented by the same point on $X$, we have found 5 $\mathbf{F}_{2}$-rational points on $X$. Therefore the Jacobian of $X$ decomposes as claimed in the statement of the theorem.

Remark 4.2. One can show that the curve $X$ has 60 cusps (that is, points that lie over the point at infinity on the $j$-line). The field of definition of 10 of the cusps is $\mathbf{F}_{2^{5}}$; the field of definition of the other 50 is $\mathbf{F}_{2^{10}}$. Using these facts, together with the modular interpretation of the noncuspidal points on $X$, we can compute the number of points on $X$ over any (reasonably small) extension of $\mathbf{F}_{2}$. This gives another method of computing the decomposition of the Jacobian of $X$. 
Remark 4.3. Applying Ligozat's Proposition 3.6.1, we see that the Jacobian of $X(11)_{K_{11}} \otimes \mathbf{F}_{3}$ splits into a product of ordinary elliptic curves. Hence the bound 26 for ordinary elliptic curves over $\mathbf{F}_{3}$ from Remark 3.7 is reached as well.

\section{Application: Modular Curves with split Jacobians}

Let $J_{0}(N)$ denote the Jacobian of the modular curve $X_{0}(N)$ over $\mathbf{Q}$. Cohen 3 . (mentioned in [15, Remarque 2, p. 90] with the value $N=27$ omitted) has computed a list of the odd integers $N$ for which $J_{0}(N)$ is isogenous to a product of elliptic curves, and Yamauchi [21, Thm. 1.1] extended this list to include even values of $N$ as well. In this section we use Theorem 3.1 and the mathematical software package Sage [17 to recompute Yamauchi's list. We note that the list in Yamauchi's theorem mistakenly includes $N=672$ and omits $N=28$.

Theorem 5.1. Let $N=2^{e} n$ with $n$ odd. Then $J_{0}(N)$ is isogenous over $\mathbf{Q}$ to a product of elliptic curves if and only if $n$ appears in the following table and $e \leq E(n)$ with $E(n)$ as tabulated:

\begin{tabular}{r|c}
$n$ & $E(n)$ \\
\hline 1 & 7 \\
3 & 7 \\
5 & 4 \\
7 & 4 \\
9 & 7 \\
11 & 2 \\
13 & 2
\end{tabular}

\begin{tabular}{r|c}
$n$ & $E(n)$ \\
\hline 15 & 4 \\
17 & 1 \\
19 & 2 \\
21 & 4 \\
25 & 4 \\
27 & 4 \\
33 & 2
\end{tabular}

\begin{tabular}{r|c}
$n$ & $E(n)$ \\
\hline 37 & 0 \\
45 & 4 \\
49 & 0 \\
57 & 1 \\
75 & 4 \\
99 & 2 \\
121 & 0
\end{tabular}

Proof. Suppose $N$ is an odd integer such that $J_{0}(N)$ splits into elliptic curves. Since $N$ is odd, the modular curve $X_{0}(N)$ has good reduction modulo 2 and the reduced curve over $\mathbf{F}_{2}$ has split Jacobian. By Theorem 3.1 the genus of $X_{0}(N)$ is at most 26. Using the fact 4 that the genus of $X_{0}(N)$ is greater than or equal to $(N-5 \sqrt{N}-8) / 12$, we find that $N$ is at most 422 .

Using the command $\mathrm{JO}(\mathrm{N})$. decomposition() of the mathematics package Sage for all $\mathrm{N}$ less than 423 , we find that the odd values of $N$ with $J_{0}(N)$ split are the twenty-one odd integers that appear as $n$ in the table.

To complete the proof, we note that if $J_{0}(N)$ is split, then so is $J_{0}(n)$ for every divisor $n$ of $N$. Therefore the integers we are searching for can be written as $2^{e} n$ for some exponent $e$ and for some $n$ among the odd values that we have just computed. For each of the possible odd parts $n$, we use Sage to compute the decomposition of $J_{0}\left(2^{e} n\right)$ for increasing values of $e$ until we reach a Jacobian that does not split. (In practice, we do not have to compute the decomposition of $J_{0}\left(2^{e} n\right)$ if we already know that $J_{0}\left(2^{e} m\right)$ does not split for some divisor $m$ of $n$. For example, since $J_{0}\left(2^{8}\right)$ does not split, we must have $E(n)<8$ for each $n$.) The largest value of $e$ for which $J_{0}\left(2^{e} n\right)$ splits is recorded in the table as $E(n)$.

Remark 5.2. Ekedahl and Serre 9] give a list of various values of $g$ such that there exists a curve of genus $g$ over $\mathbf{Q}$ with a completely split Jacobian. Many of their values of $g$ come from modular curves $X_{0}(N)$. The tables of modular forms that they had access to did not include values of $N$ greater than 1000 , so they missed a few of the values from Theorem 5.1. The modular curves $X_{0}(1152)$ and $X_{0}(1200)$, of genus 161 and 205, respectively, allow us to add two more values of $g$ to their list [9, Théorème, p. 509]. 
Ekedahl and Serre also seem to have missed the curve $X_{0}(396)$ of genus 61 , but they obtain a curve of genus 61 with split Jacobian by considering a quotient of $X_{0}(720)$ by an involution.

\section{ACKNOWLEDGMENTS}

After an initial version of this work was posted on the arXiv, Mike Zieve alerted us to the paper of Duursma and Enjalbert [8] mentioned above, which contains a result that implies our Lemma 2.1 (see $\S 2.4$ of the arXiv version of [8]) and which proves that a curve over $\mathbf{F}_{2}$ whose Jacobian is isogenous to a product of elliptic curves has genus at most 26 . The print version of their paper also states that there is a model of $X(11)$ over $\mathbf{F}_{2}$ whose Jacobian is isogenous over $\mathbf{F}_{2}$ to a product of elliptic curves; this is proved in an addendum added to the arXiv version. We are grateful to Mike Zieve for telling us about [8] and to Iwan Duursma for discussions about these results.

We are grateful to Armand Brumer for informing us of Yamauchi's paper [21.

In the initial version of this paper, the bound $B_{1}$ in Theorem 1.1 was a doubly exponential expression in $s$ that we obtained via an argument using our Lemma 2.1. We are extremely grateful to Zeev Rudnick and Sergei Konyagin for pointing out to us that the work of Smyth [16] could be used to get a bound that is singly exponential in $s$. Our proof that $B_{1}$ gives an upper bound for the genus is due almost entirely to them.

The work in this paper was begun at the GeoCrypt 2009 conference in Pointeà-Pitre. We would like to thank the organizers of the conference for providing a stimulating environment for collaboration.

\section{REFERENCES}

[1] Peter Bending, Alan Camina, and Robert Guralnick, Automorphisms of the modular curve, Progress in Galois theory, Dev. Math., vol. 12, Springer, New York, 2005, pp. 25-37, DOI 10.1007/0-387-23534-5_2. MR2148458 (2006c:14050)

[2] Wieb Bosma, John Cannon, and Catherine Playoust, The Magma algebra system. I. The user language, Computational algebra and number theory (London, 1993), J. Symbolic Comput. 24 (1997), no. 3-4, 235-265, DOI 10.1006/jsco.1996.0125. MR1484478

[3] Henri Cohen, Sur les $N$ tels que $J_{0}(N)$ soit $\mathbf{Q}$-isogène à un produit de courbes elliptiques. Undated preprint, available at http://www.math.u-bordeaux1.fr/ cohen/serretrace.dvi.

[4] János A. Csirik, Joseph L. Wetherell, and Michael E. Zieve, On the genera of $X_{0}(N)(2000)$. http://arxiv.org/abs/math.NT/0006096, arXiv:math/0006096v2 [math.NT].

[5] Max Deuring, Die Typen der Multiplikatorenringe elliptischer Funktionenkörper, Abh. Math. Sem. Hansischen Univ. 14 (1941), 197-272 (German). DOI 10.1007/BF02940746. MR0005125 $(3,104 \mathrm{f})$

[6] Stephen A. DiPippo and Everett W. Howe, Real polynomials with all roots on the unit circle and abelian varieties over finite fields, J. Number Theory 73 (1998), no. 2, 426-450, DOI 10.1006/jnth.1998.2302. MR.1657992(2000c:11101)

[7] Stephen A. DiPippo and Everett W. Howe, Corrigendum: "Real polynomials with all roots on the unit circle and abelian varieties over finite fields" [J. Number Theory 73 (1998), no. 2, 426-450; MR1657992 (2000c:11101)], J. Number Theory 83 (2000), no. 1, 182, DOI 10.1006/jnth.2000.2565. MR.1767658

[8] Iwan Duursma and Jean-Yves Enjalbert, Bounds for completely decomposable Jacobians, Finite fields with applications to coding theory, cryptography and related areas (Oaxaca, 2001), Springer, Berlin, 2002, pp. 86-93. Electronic version, with an addendum, available at arXiv:1007.3344v1 [math.NT]. MR1995329(2005d:14032) 
[9] Torsten Ekedahl and Jean-Pierre Serre, Exemples de courbes algébriques à jacobienne complètement décomposable, C. R. Acad. Sci. Paris Sér. I Math. 317 (1993), no. 5, 509513 (French, with English and French summaries). MR 1239039 (94j:14029)

[10] Daniel Goldstein, Robert M. Guralnick, Everett W. Howe, and Michael E. Zieve, Nonisomorphic curves that become isomorphic over extensions of coprime degrees, J. Algebra 320 (2008), no. 6, 2526-2558, DOI 10.1016/j.jalgebra.2008.06.003. MR2437513 (2009h:14051)

[11] Yasutaka Ihara, Some remarks on the number of rational points of algebraic curves over finite fields, J. Fac. Sci. Univ. Tokyo Sect. IA Math. 28 (1981), no. 3, 721-724 (1982). http://hdl.handle.net/2261/6319. MR656048 (84c:14016)

[12] Gérard Ligozat, Courbes modulaires de niveau 11, Modular functions of one variable, V (Proc. Second Internat. Conf., Univ. Bonn, Bonn, 1976), Lecture Notes in Math., vol. 601, Springer, Berlin, 1977, pp. 149-237 (French). DOI 10.1007/BFb0063948. MR0463118 (57 \#3079)

[13] Christophe Ritzenthaler, Automorphismes des courbes modulaires $X(n)$ en caractéristique p, Manuscripta Math. 109 (2002), no. 1, 49-62 (French, with English summary), DOI 10.1007/s002290200286. MR:1931207 (2003g:11067)

[14] Hans-Georg Rück and Henning Stichtenoth, A characterization of Hermitian function fields over finite fields, J. Reine Angew. Math. 457 (1994), 185-188, DOI 10.1515/crll.1994.457.185. MR:1305281 (95h:11059)

[15] Jean-Pierre Serre, Répartition asymptotique des valeurs propres de l'opérateur de Hecke $T_{p}$, J. Amer. Math. Soc. 10 (1997), no. 1, 75-102 (French), DOI 10.1090/S0894-0347-97-00220-8. MR 1396897 (97h:11048)

[16] C. J. Smyth, Some inequalities for certain power sums, Acta Math. Acad. Sci. Hungar. 28 (1976), no. 3-4, 271-273. DOI 10.1007/BF01896789. MR0424710 (54 \#12669)

[17] William Stein et al., Sage Mathematics Software (Version 4.0.1), the Sage Development Team, 2009. http://www.sagemath.org/.

[18] Michael A. Tsfasman, Some remarks on the asymptotic number of points, Coding theory and algebraic geometry (Luminy, 1991), Lecture Notes in Math., vol. 1518, Springer, Berlin, 1992, pp. 178-192, DOI 10.1007/BFb0088001. MR1186424 (93h:11064)

[19] M. A. Tsfasman and S. G. Vlăduț, Asymptotic properties of zeta-functions, Algebraic geometry, 7, J. Math. Sci. (New York) 84 (1997), no. 5, 1445-1467, DOI 10.1007/BF02399198. MR:1465522(98h:11079)

[20] William C. Waterhouse, Abelian varieties over finite fields, Ann. Sci. École Norm. Sup. (4) 2 (1969), 521-560. http://www.numdam.org/item?id=ASENS_1969_4_2_4_521_0. MR0265369 (42\#279)

[21] Takuya Yamauchi, On $\mathbb{Q}$-simple factors of Jacobian varieties of modular curves, Yokohama Math. J. 53 (2007), no. 2, 149-160. MR2302608 (2008k:11062)

Department of Mathematics, Harvard University, Cambridge, Massachusetts 021382901

E-mail address: elkies@math.harvard.edu

URL: http://www.math.harvard.edu/ elkies/

Center for Communications Research, 4320 Westerra Court, San Diego, California 92121-1967

E-mail address: however@alumni.caltech.edu

URL: http: //www . alumni.caltech.edu/ however/

Institut de Mathématiques de Luminy, UMR 6206 du CNRS, Luminy, Case 907, 13288 Marseille, France

E-mail address: ritzenth@iml.univ-mrs.fr

$U R L:$ http://iml.univ-mrs.fr/ ritzenth/ 